\title{
Assessment of the serial changes of vessel wall contents in atherosclerotic coronary lesion with bioresorbable everolimus-eluting vascular scaffolds using Shin's method: an IVUS study
}

\author{
Eun-Seok Shin · Hector M. Garcia-Garcia - Scot Garg • \\ Jurgen Ligthart • Leif Thuesen · Dariusz Dudek • \\ John A. Ormiston · Patrick W. Serruys
}

Received: 8 July 2010/ Accepted: 21 October 2010

(C) Springer Science+Business Media, B.V. 2010

\begin{abstract}
Although serial changes in necrotic core and calcium are regarded as surrogates for the bioresorption process in patients treated with the bioresorbable everolimus-eluting vascular scaffolds (BVS), these temporal changes have not yet been fully investigated. Shin's method may be offer a more suitable technique for this analysis because it includes all the contents of both the lumen and vessel wall. The purpose of this study was to assess the serial changes of necrotic core and dense calcium content in coronary lesions that were treated with a BVS implant using Virtual Histology intravascular ultrasound (VH-IVUS) analyzed using Shin's method. A total of 29 patients (92 coronary segments) were imaged to evaluate the serial
\end{abstract}

\section{E.-S. Shin}

Ulsan University Hospital, University of Ulsan College of Medicine, Ulsan, Korea

E.-S. Shin · H. M. Garcia-Garcia - S. Garg ·

J. Ligthart · P. W. Serruys $(\bowtie)$

Thoraxcenter, Erasmus MC, Bd 585, 's-Gravendijkwal

230, 3015-CE Rotterdam, The Netherlands

e-mail: p.w.j.c.serruys@erasmusmc.nl

L. Thuesen

Skejby Sygehus, Aarhus University Hospital, Skejby,

Denmark

D. Dudek

Jagiellonian University, Krakov, Poland

J. A. Ormiston

Auckland City Hospital, Auckland, New Zealand changes in necrotic core and dense calcium using Shin's method. Lesions treated with a BVS implant were analyzed with serial VH-IVUS assessments, i.e., pre- and post-stenting, and at 6 months and 2 years follow-up. In Shin's method contours are drawn around the IVUS catheter (instead of delineating the lumen) and the vessel. The mean necrotic core area decreased by $6.9 \%$ from post-stenting to 6 months (1.71 \pm $1.03 \mathrm{~mm}^{2}$ vs. $\left.1.36 \pm 0.91 \mathrm{~mm}^{2}, P=0.027\right)$, and by $20.5 \% \quad\left(1.71 \pm 1.03 \mathrm{~mm}^{2}\right.$ vs. $1.20 \pm 0.70 \mathrm{~mm}^{2}$, $P=0.003)$ from post-steting to 2 years; while the mean dense calcium areas decreased by $27.2 \%$ $\left(1.07 \pm 0.55 \mathrm{~mm}^{2}\right.$ vs. $\left.0.78 \pm 0.64 \mathrm{~mm}^{2}, P=0.039\right)$ from post-stenting and 2 years. At 2 years, absolute necrotic core and dense calcium content were significantly decreased as compared to post-stenting values. The present study demonstrates that the bioresorption process in patients who undergoing BVS device implantation can be assessed using VH-IVUS analysed using Shin's method.

Keywords Virtual Histology intravascular ultrasound · Bioabsorbable stent · Bioresorption

\section{Introduction}

Bioresorbable scaffolds have the potential to reduce adverse events such as stent thrombosis (ST) when compared to conventional metallic stents, because 
drug elution and vessel scaffolding are only provided by the implant until the vessel has healed, and as such no longer triggers for ST, such as non-endothelialized stent struts, or drug polymers are present during long time [1]. In several studies, of patients undergoing implantation of the bioresorbable everolimus-eluting vascular scaffolds (BVS: Abbott Vascular, Santa Clara, CA), necrotic core and dense calcium assessed using Virtual Histology intravascular ultrasound (VH-IVUS) have been used as surrogates to monitor the bioresorption process. This came about following the observed abrupt increase in necrotic core and dense calcium seen immediately after BVS implantation, and the gradually diminished levels seen with follow-up. In these previous reports, the bioresorption process has been explored using conventional $\mathrm{VH}-$ IVUS analysis [1,2]. The accuracy of this conventional analysis however, may have been affected because some BVS strut information may have been missed due to the presence of a medial grey stripe (which is arbitrary delineated in the color frames of VH-IVUS and represents the location of the media layer of the vessel wall), and also because of temporal changes in the appearance of the BVS strut which might have precluded proper contouring. These pertinent issues can be overcome by analysing the VH-IVUS using Shin's method, which includes all the content of the lumen and vessel wall, and has a high reproducibility [3], thereby allowing complete characterization of serial changes in composition within the external elastic membrane and the catheter.

The purpose of this study was to assess whether serial changes in "necrotic core" and "dense calcium" content assessed using VH-IVUS (these are artefacts since VH-IVUS has not been validated for this use, therefore we will write them between quotation marks) and analysed using a new method (Shin's method).

\section{Methods}

Study patients

The study design for the ABSORB Cohort A trial has been published elsewhere [4]. VH-IVUS images were obtained from 29 out the 30 patients participating in the ABSORB Cohort A trial who treated with the
BVS implant; one patient was excluded because no follow-up images were available. The study protocol was approved by the institutional ethics committee, and written informed consent was obtained from all patients.

\section{VH-IVUS acquisition and examination}

In brief, IVUS data were acquired with commercially available phased-array IVUS catheters (Eagle Eye Gold 2.9-F $20 \mathrm{MHz}$, Volcano Corporation, Rancho Cordova, USA) by a dedicated VH-IVUS console (Volcano Therapeutics, Rancho Cordova, USA). Four major components (fibrous: green; fibrofatty: lightgreen; dense calcium: white; and necrotic core: red) were characterized and image acquisition was ECGgated. After intracoronary injection of $200 \mu \mathrm{g}$ nitroglycerine, continuous pullback of the IVUS catheter was performed using a motorized pullback device at $0.5 \mathrm{~mm} / \mathrm{s}$. Data of both pullbacks were stored on hard disk for off-line analysis. VH-IVUS analysis was performed by one experienced IVUS analyst (E.S.S.) in an independent core laboratory (Cardialysis BV, Rotterdam, The Netherlands) using pcVH 2.2 off-line software.

\section{Procedures}

In Shin's method, instead of measuring the lumen as previously described in the conventional method of VH-IVUS analysis, contours around the IVUS catheter are drawn without following the leading edge of the interface lumen intima. According to Shin's method, when there is a "flare" (ring-down artifact) around the IVUS catheter, this catheter contour must be drawn away from this flare. The catheter contour was manually detected. In addition, to avoid coverage of scaffold struts by the superimposed medial stripe, the vessel contour was drawn purposefully slightly outwards from the struts but without including the adventitia (Fig. 1).

On each cross section, polymeric scaffold struts were detected as areas of apparent "dense calcium" surrounded by "necrotic core". We used the change in quantitative analysis of these areas between implantation and follow-up as a surrogate assessment of the bioresorption process. In addition, we analyzed the relative amount of "necrotic core and dense 

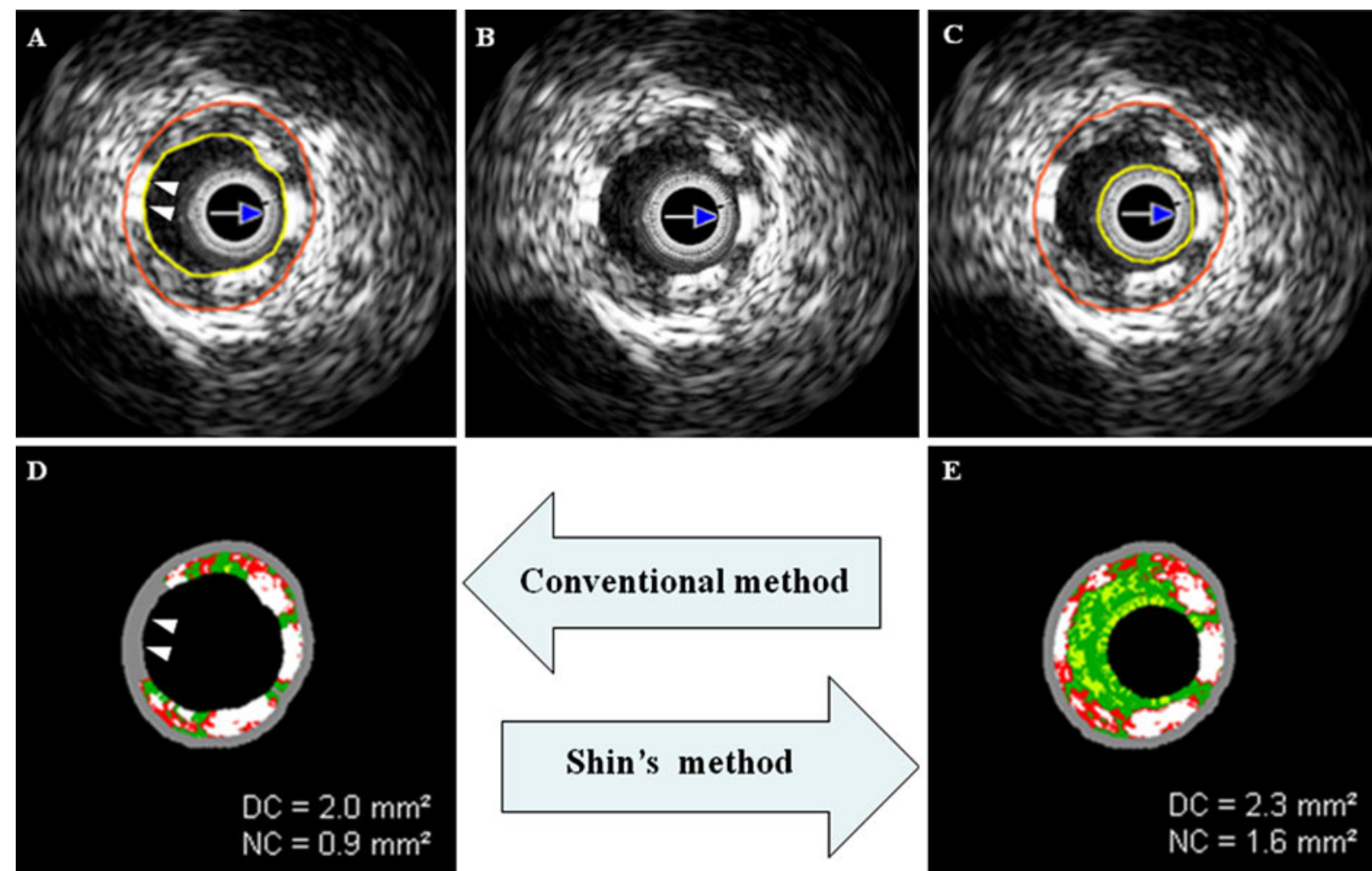

Fig. 1 To avoid coverage of stent struts by the superimposed medial stripe, the vessel contour was drawn purposefully slightly outwards from the struts but without including the adventitia. Shin's method shows some "necrotic core and

calcium" with vessel volume to compare serial changes in the percentage for "necrotic core and dense calcium".

\section{Experimental study}

In a harvested pig coronary artery flushed continuously with saline solution at $37^{\circ} \mathrm{C}$, a VH-IVUS pullback was performed prior to any intervention. ECG gating was simulated using an external source. Next, a BVS device $(3.0 \times 18 \mathrm{~mm})$ was implanted at nominal pressure and immediately a second $\mathrm{VH}-$ IVUS pullback was performed.

The aim for this experimental study was to compare the change of "necrotic core and dense calcium" after stenting measured by Shin's method between harvested pig coronary artery and Absorb trial, Cohort A with the delta value of "necrotic core and dense calcium" before and after BVS implantation by Cohort A. dense calcium" for the bioabsorbable everolimus-eluting stent in the grey medial stripe (c and e) that was not shown with the conventional method (a and $\mathbf{d}$; two white arrow heads) (DC dense calcium, $N C$ necrotic core)

Statistical analysis

Analyses were performed with SPSS 16.0 (SPSS Inc., Chicago, Illinois). Discrete variables are presented as counts and percentages. Continuous variables are presented as means $\pm 1 \mathrm{SD}$. Serial changes of "necrotic core and dense calcium" were compared using paired Student's $t$ test. Nonparametric analysis with Wilcoxon signed rank test was also done. A twosided $P$ value of less than 0.05 indicated statistical significance.

\section{Results}

Patient characteristics are shown in Table 1. The mean patient age was 62 years, and 18 participants were male. The study vessels were the left anterior descending artery in 15, the left circumflex artery in 9 and the right coronary artery in 7 cases. 
Table 1 Baseline characteristics

\begin{tabular}{ll}
\hline & $\mathrm{N}=30$ \\
\hline Age, years & $62 \pm 9$ \\
Male & $18(60)$ \\
Diabetes & $1(3)$ \\
Hypertension & $18(60)$ \\
Current smoker & $6(20)$ \\
Hypercholesterolemia & $19(63)$ \\
Study vessel & \\
LAD & $15(47)$ \\
LCX & $9(30)$ \\
RCA & $7(23)$ \\
Lesion length, mm & $12.83 \pm 3.74$ \\
\hline
\end{tabular}

Values are presented as number $(\%)$ or mean $\pm \mathrm{SD}, L A D$ left anterior descending artery, $L C X$ left circumflex artery, $R C A$ right coronary artery

Serial change of "necrotic core and dense calcium"

Table 2 shows serial changes in "necrotic core", "dense calcium" and the mean vessel area and volume (external elastic membrane), After stenting, "necrotic core and dense calcium" content increased because BVS struts were characterized as "dense calcium" with large quantities of "necrotic core" surrounding them. Overall, after stenting there was a gradual reduction during follow-up in mean vessel area, "necrotic core" area and "dense calcium" area (mean vessel area: $14.1 \pm 3.5,13.9 \pm 3.4$, $12.8 \pm 3.6 \mathrm{~mm}^{2}$, mean "necrotic core" area: $1.71 \pm 1.03,1.36 \pm 0.91,1.20 \pm 0.70 \mathrm{~mm}^{2}$, mean "dense calcium" area: $1.07 \pm 0.55,0.85 \pm 0.54$, $0.78 \pm 0.64 \mathrm{~mm}^{2}$ at post-stenting, 6 months and 2 years follow-up, respectively). From post-stenting to 6 months, the mean "necrotic core" area decreased significantly $(-6.9 \pm 55.6 \%, P=0.027)$. From poststenting to 2 years, mean "necrotic core and dense calcium" areas decreased also significantly $(-20.5 \pm 33.6 \%, P=0.003$ and $-27.2 \pm 48.8 \%$, $P=0.039$, respectively) (Fig. 2). In volumetric analysis, from post-stenting to 2 years, "necrotic core" volume decreased significantly $(-16.4 \pm$ $42.1 \%, P=0.023$ ) whilst a trend was seen for a reduction in "dense calcium" $(-22.0 \pm 58.1 \%$, $P=0.057)$.

\section{Experimental results}

In the harvested pig coronary artery, there was an immediate increase in "necrotic core and dense calcium" areas after BVS deployment, which is comparable to the increase observed following BVS implantation in the ABSORB cohort A study (Table 3).

Table 2 Serial changes of mean area and volume of vessel (EEM), "necrotic core and dense calcium" in pre- and post-stenting, and at 6 months and 2 years follow-up

\begin{tabular}{|c|c|c|c|c|c|c|}
\hline & \multicolumn{3}{|c|}{ Mean area $\left(\mathrm{mm}^{2}\right)$} & \multicolumn{3}{|l|}{ Volume $\left(\mathrm{mm}^{3}\right)$} \\
\hline & EEM & $\mathrm{NC}$ & $\mathrm{DC}$ & EEM & $\mathrm{NC}$ & $\mathrm{DC}$ \\
\hline Pre-stenting $(n=14)$ & $13.2 \pm 3.5$ & $0.87 \pm 0.73$ & $0.39 \pm 0.40$ & $180.6 \pm 64.2$ & $12.1 \pm 11.0$ & $5.4 \pm 5.9$ \\
\hline Post-stenting $(n=28)$ & $14.1 \pm 3.5$ & $1.71 \pm 1.03$ & $1.07 \pm 0.55$ & $173.1 \pm 60.6$ & $21.5 \pm 13.7$ & $13.3 \pm 7.5$ \\
\hline 6 months $(n=28)$ & $13.9 \pm 3.4$ & $1.36 \pm 0.91$ & $0.85 \pm 0.54$ & $192.0 \pm 98.0$ & $19.4 \pm 16.4$ & $11.6 \pm 7.5$ \\
\hline 2 years $(n=22)$ & $12.8 \pm 3.6$ & $1.20 \pm 0.70$ & $0.78 \pm 0.64$ & $163.3 \pm 72.6$ & $15.5 \pm 10.5$ & $10.2 \pm 8.4$ \\
\hline $\begin{array}{l}\text { Relative } \triangle(\%) \text { post-stenting } \\
\text { vs. } 6 \text { months }\end{array}$ & $-1.5 \pm 8.8$ & $-6.9 \pm 55.6$ & $-5.9 \pm 63.1$ & $6.5 \pm 32.0$ & $-3.1 \pm 53.7$ & $-1.9 \pm 60.8$ \\
\hline $\begin{array}{l}\text { Relative } \triangle(\%) 6 \text { months } \\
\text { vs. } 2 \text { years }\end{array}$ & $-4.6 \pm 7.8$ & $-7.7 \pm 49.9$ & $-7.7 \pm 70.6$ & $-0.3 \pm 39.3$ & $-1.8 \pm 62.3$ & $-4.3 \pm 74.1$ \\
\hline $\begin{array}{l}\text { Relative } \triangle(\%) \text { post-stenting } \\
\text { vs. } 2 \text { years }\end{array}$ & $-6.7 \pm 13.4$ & $-20.5 \pm 33.6$ & $-27.2 \pm 48.8$ & $-3.3 \pm 30.3$ & $-16.4 \pm 42.1$ & $-22.0 \pm 58.1$ \\
\hline$P$ value post-stenting vs. 6 months & 0.268 & 0.027 & 0.081 & 0.240 & 0.338 & 0.198 \\
\hline$P$ value 6 months vs. 2 years & 0.065 & 0.162 & 0.363 & 0.432 & 0.250 & 0.404 \\
\hline$P$ value post-stenting vs. 2 years & 0.058 & 0.003 & 0.039 & 0.327 & 0.023 & 0.057 \\
\hline
\end{tabular}

Values are presented as mean $\pm \mathrm{SD}, E E M$ external elastic membrane, $N C$ necrotic core, $D C$ dense calcium, $\triangle$ difference 

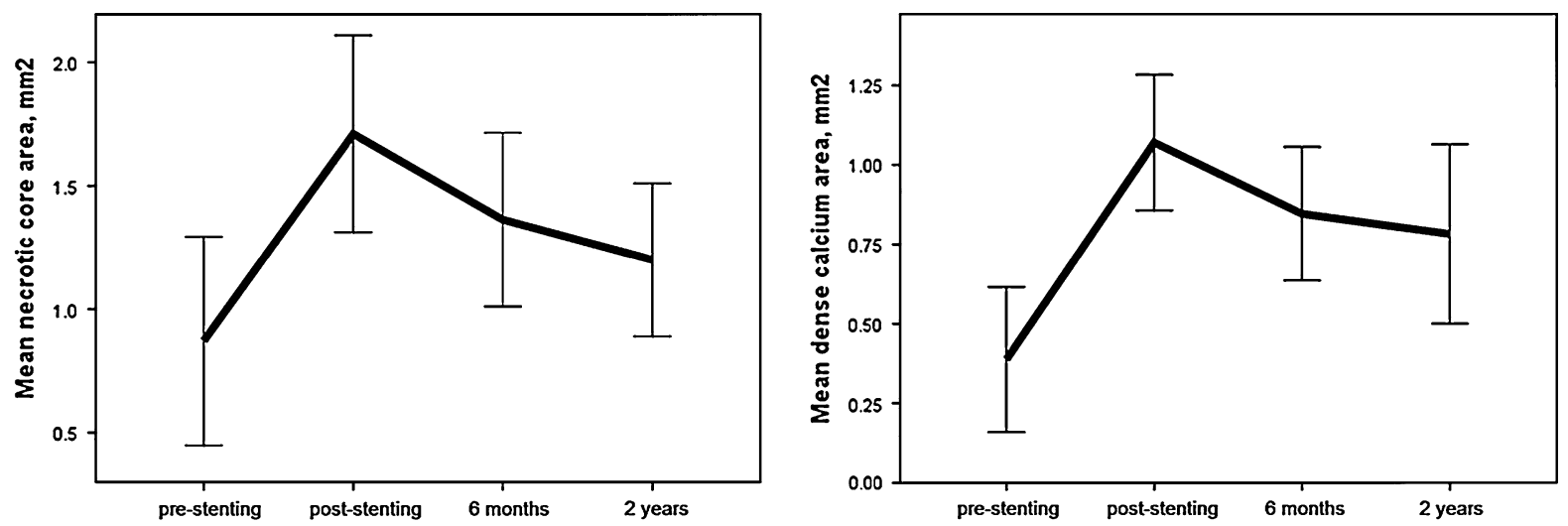

Fig. 2 Serial changes in mean areas of "necrotic core and dense calcium" (display error bars $=95 \%$ Confidence intervals)

Table 3 Absolute differences in "necrotic core and dense calcium" areas following BVS implantation in pig and human coronary arteries

\begin{tabular}{llc}
\hline & \multicolumn{2}{l}{$\begin{array}{l}\text { Absolute difference between } \\
\text { pre- and post-stenting }\end{array}$} \\
\cline { 2 - 3 } & $\begin{array}{l}\text { BVS in harvested pig } \\
\text { coronary artery }(\mathrm{N}=1)\end{array}$ & $\begin{array}{c}\text { Absorb cohort } \\
\text { A }(\mathrm{N}=12)\end{array}$ \\
\hline Stent area $\left(\mathrm{mm}^{2}\right)$ & $2.43 \pm 0.49$ & $2.49 \pm 0.78$ \\
NC area $\left(\mathrm{mm}^{2}\right)$ & $1.37 \pm 0.25$ & $1.60 \pm 0.64$ \\
DC area $\left(\mathrm{mm}^{2}\right)$ & $1.06 \pm 0.28$ & $0.90 \pm 0.39$ \\
$\%$ NC to vessel area & $8.9 \pm 1.6$ & $8.7 \pm 2.6$ \\
$\%$ DC to vessel area & $6.9 \pm 1.9$ & $5.1 \pm 2.1$ \\
\hline
\end{tabular}

Values are presented as mean $\pm \mathrm{SD}, N C$ necrotic core, $D C$ dense calcium, Stent area $=$ post-stenting $\mathrm{NC}$ and DC areas minus pre-stenting $\mathrm{NC}$ and DC areas

\section{Discussion}

The main finding of this study is that the mean area of "necrotic core and dense calcium" as assessed using Shin's method decreased significantly between baseline and 2-years follow-up in the lesion of BVS implantation. The serial changes in absolute volumes of "necrotic core and dense calcium" seen on VHIVUS analyzed using Shin's method, can be used as a surrogate marker of the bioresorption process in coronary lesions treated with the BVS implant.

Biodegradable stents offers several distinct advantages over conventional bare or drug coated metallic stents. These include potential reductions in adverse events such as ST, and a reduced requirement for long-term dual anti-platelet therapy as foreign materials could be decreased at long-term. Physiologically, the absence of a rigid metallic casing can facilitate the return of vessel vasomotion, adaptive shear stress, late luminal enlargement and late expansive remodeling. Additional long-term advantages include an improvement in future treatment options, as percutaneous coronary intervention or surgical revascularization can be performed in areas of previous stenting without restriction. Furthermore, biodegradable stents can negate some of the other problems associated with use of permanent metallic stents such as the covering of side branches, overhang at ostial lesions, and the 'blooming effect' seen when using non-invasive imaging techniques such as CT angiography or MRI [5].

Biodegradable stents are currently undergoing clinical trials, and currently the BVS implant which represents the most extensively studied device, remains the most important source of knowledge regarding the bioresorption process in vivo. In the ABSORB study implanted BVS polymeric struts appeared as highly echogenic/calcific structures on VH-IVUS (Fig. 1). After device implantation an increase in "dense calcium" with surrounding "necrotic core" was observed. This has been attributed to the polymeric struts, and therefore "dense calcium" with surrounding "necrotic core" has been suggested as the $\mathrm{VH}$ fingerprint of polymeric struts [2]. This hypothesis is supported by the results of our experimental study in which the recorded increase in "necrotic core" area and "dense calcium" area was similar to that observed in Absorb cohort A. Although several previous papers with the same patient cohort reported a decrease in the relative amounts of "necrotic core and dense calcium", the 
absolute amount of "necrotic core and dense calcium" failed to show statistically significant decrease [2] except for "necrotic core" area at 2 years [1]. In the present study, at 2 years, mean area of "necrotic core" shrank by $20.5 \%$, whilst mean area of "dense calcium" decreased by $27.2 \%\left(1.07 \pm 0.55 \mathrm{~mm}^{2}\right.$ at post-stenting vs. $0.78 \pm 0.64 \mathrm{~mm}^{2}$ at 2 years).

In a recent study, we introduced a new catheter contour based method of measuring necrotic core and dense calcium (Shin's method), in contrast to the conventional lumen contour based one. We have previously demonstrated that Shin's method has a good correlation with conventional methods for the calculation of necrotic core and dense calcium, with shorter measuring times and excellent reproducibility in both stented and non-stented lesions [3]. In longitudinal studies, accurate assessment must take into account the variability in: IVUS catheters, analyst and method of analysis. For example, in a previous study the inter-catheter variability was 6-8\% for necrotic core and dense calcium when analyzed by the same observer [6]. In a similar assessment using Shin's method the relative differences in necrotic core and dense calcium were only $1 \%$ in intra-observer comparisons (Shin et al., submitted). Therefore Shin's method can be used in order to minimize the impact of the other factors that contribute to variability in longitudinal assessments.

Although the bioresorption process has been previously explored using VH-IVUS in human coronary arteries [2], some BVS strut information may have been missed because of the presence of the medial grey stripe (Fig. 1), and/or because of temporal changes in the BVS strut appearance which might have precluded proper contouring (i.e., struts in the vicinity of a calcified area are hard to discriminate from calcium). Shin's method draws a catheter contour (instead of a lumen contour), thereby including all contents of the lumen and vessel wall, thus allowing a complete analysis of serial changes in plaque composition, (i.e., necrotic core and dense calcium) which can be used as a surrogate of the bioresorption process (Fig. 3). Therefore Shin's method achieves a more complete characterization of all components within the vessel.

\section{Limitations}

This study had several limitations. (1) The sample size is small and therefore, serial changes in "necrotic core and dense calcium" content should be interpreted cautiously. (2) VH-IVUS has not been

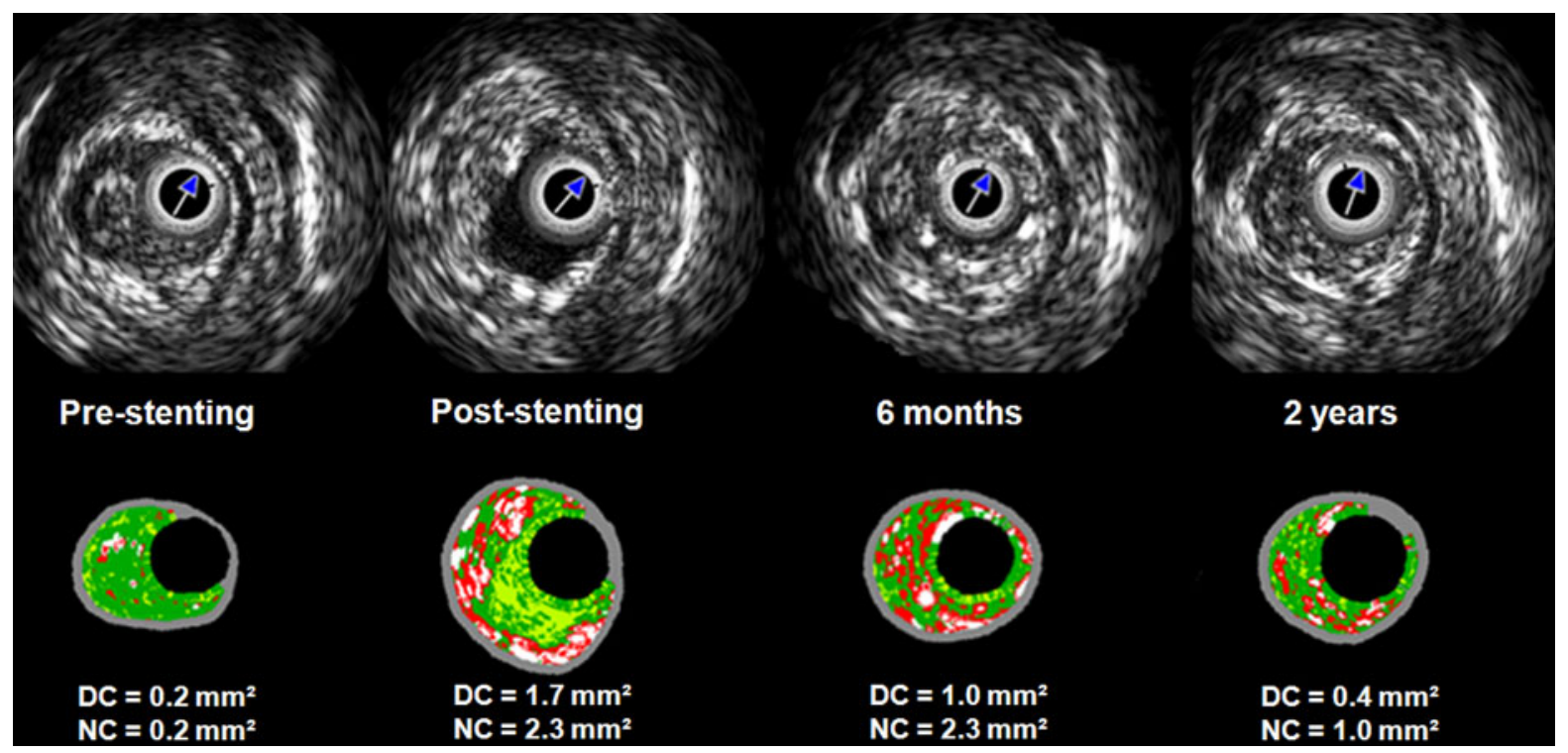

Fig. 3 Serial assessment using Shin's method by Virtual Histology intravascular ultrasound. Pre-stenting, there is a large plaque. After stenting, the lumen has enlarged and stent struts are depicted as "necrotic core with dense calcium". At
6 months, the stent struts remained visible with partial reduction of the luminal area. At 2 years, the endoluminal "dense calcium" depicting struts has decreased, which is consistent with strut absorption. ( $N C$ necrotic core, $D C$ dense calcium) 
specifically validated for the assessment of polymeric struts in vitro and ex vivo. In addition, the "necrotic core and dense calcium" seen after the implantation of BVS device are artefacts and not real tissue types, since virtual histology has never been validated for this use. (3) It is difficult to assess, if not impossible, whether plaque contents have progressed or regressed after complete resorption of stent strut at follow-up in this study. For instance, if the plaque progressed at 2 year follow-up, there is a possibility for "necrotic core with dense calcium" to be bigger, in contrast, if the plaque regressed at 2 year follow-up, there is a possibility for "necrotic core with dense calcium" to be smaller.

\section{Conclusions}

At 2 years, absolute "necrotic core and dense calcium" content were significantly decreased as compared to post-stenting values. The present study demonstrates that the bioresorption process in patients who undergoing BVS device implantation can be assessed using VH-IVUS analysed using Shin's method.

Conflict of interest We declare that there is no conflict of interest for any author.

\section{References}

1. Serruys PW, Ormiston JA, Onuma Y et al (2009) A bioabsorbable everolimus-eluting coronary stent system (ABSORB): 2-year outcomes and results from multiple imaging methods. Lancet 373:897-910

2. Garcia-Garcia HM, Gonzalo N, Pawar R et al (2009) Assessment of the absorption process following bioabsorbable everolimus-eluting stent implantation: temporal changes in strain values and tissue composition using intravascular ultrasound radiofrequency data analysis. A substudy of the ABSORB clinical trial. EuroIntervention 4:443-448

3. Shin ES, Garcia-Garcia HM, Serruys PW (2010) A new method to measure necrotic core and calcium content in coronary plaques using intravascular ultrasound radiofrequency-based analysis. Int J Cardiovasc Imaging 26: 387-396

4. Ormiston JA, Serruys PW, Regar E et al (2008) A bioabsorbable everolimus-eluting coronary stent system for patients with single de novo coronary artery lesions (ABSORB): a prospective open-label trial. Lancet 371: 899-907

5. Spuentrup E, Ruebben A, Mahnken A et al (2005) Artifactfree coronary magnetic resonance angiography and coronary vessel wall imaging in the presence of a new, metallic, coronary magnetic resonance imaging stent. Circulation 111:1019-1026

6. Rodriguez-Granillo GA, Vaina S, Garcia-Garcia HM et al (2006) Reproducibility of intravascular ultrasound radiofrequency data analysis: implications for the design of longitudinal studies. Int J Cardiovasc Imaging 22:621-631 